\title{
Observation of magnetic susceptibility changes within the thalamus: a comparative study between healthy and Parkinson's disease afflicted cynomolgus monkeys using $7 \mathrm{~T}$ MRI
}

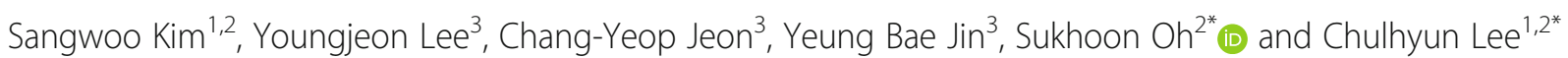

\begin{abstract}
Background: Although the thalamus is known to modulate basal ganglia function related to motor control activity, the abnormal changes within the thalamus during distinct medical complications have been scarcely investigated. In order to explore the feasibility of assessing iron accumulation in the thalamus as an informative biomarker for Parkinson's disease (PD), this study was designed to employ quantitative susceptibility mapping using a $7 \mathrm{~T}$ magnetic resonance imaging system in cynomolgus monkeys. A 1-methyl-4-phenyl-1,2,3,6-tetrahydropyridineinjected cynomolgus monkey and a healthy control $(\mathrm{HC})$ were examined by $7 \mathrm{~T}$ magnetic resonance imaging. Positron emission tomography with ${ }^{18} \mathrm{~F}-\mathrm{N}$-(3-fluoro propyl)-2ß-carboxymethoxy-3ß-(4-iodophenyl) nortropane was also employed to identify the relationship between iron deposits and dopamine depletion. All acquired values were averaged within the volume of interest of the nigrostriatal pathway.

Findings: Compared with the HC, the overall elevation of iron deposition within the thalamus in the Parkinson's disease model (about 53.81\% increase) was similar to that in the substantia nigra (54.81\%) region. Substantial susceptibility changes were observed in the intralaminar part of the thalamus (about $70.78 \%$ increase). Additionally, we observed that in the Parkinson's disease model, binding potential values obtained from positron emission tomography were considerably decreased in the thalamus (97.51\%) and substantia nigra (92.48\%).

Conclusions: The increased iron deposition in the thalamus showed negative correlation with dopaminergic activity in PD, supporting the idea that iron accumulation affects glutaminergic inputs and dopaminergic neurons. This investigation indicates that the remarkable susceptibility changes in the thalamus could be an initial major diagnostic biomarker for Parkinson's disease-related motor symptoms.
\end{abstract}

Keywords: Parkinson's disease, Magnetic susceptibility, Ultra-high field, 7 T magnetic resonance imaging

\footnotetext{
* Correspondence: sukhoonoh@kbsi.re.kr; chulhyun@kbsi.re.kr

${ }^{2}$ Bioimaging Research Team, Korea Basic Science Institute, 162,

Yeongudanji-ro, Ochang-eup, Cheongwon-gu, Cheongju-si,

Chungcheongbuk-do, Republic of Korea

'Department of Bio-Analytical Science, University of Science and Technology,

217, Gajeong-ro, Yuseong-gu, Daejeon, Republic of Korea

Full list of author information is available at the end of the article
} 


\section{Introduction}

Parkinson's disease (PD) is one of the most common neurodegenerative diseases, characterized by progressive and severe motor failures such as resting tremor, bradykinesia, and rigidity ( $\mathrm{Xu}$ et al. 2017; Langkammer et al. 2016; Zhao et al. 2017; Murakami et al. 2015; Alkemade et al. 2017). The etiology of this disease is related to activity changes in dopaminergic neurons of the nigrostriatal pathway (NSP) (Lee et al. 2011) that consists of multiple structures including the substantia nigra (SN), globus pallidus (GP), putamen (PT), caudate nucleus $(\mathrm{CN})$, and thalamus (TH) (Hodaie et al. 2007). In PD, the loss of dopaminergic neurons is mostly concentrated in the SN pars compacta, followed by a secondary change in the dorsal striatum (combined region of the GP and PT) (Xu et al. 2017; Guan et al. 2017). The dopamine depletion in the striatum is involved in the corticostriatal dysfunction of motor circuits within the basal ganglia, which increases the activation of the indirect pathway and decreases that of the direct pathway, thereby contributing to the PD-related motor symptoms (Albin et al. 1989; DeLong 1990). In contrast, an interesting concept proposed that $\mathrm{TH}$ neurons themselves regulate basal ganglia activity by dynamically altering the signals received by motor cortical regions (Halliday 2009). A recent study has found that in the absence of corticostriatal changes in PD mice models, synaptic disorders of the thalamostriatal pathway show identical motor-modified mechanisms (Parker et al. 2016), as mentioned above. Hence, various investigations on the thalamic abnormality are needed for a better understanding of PD pathology.

Subtle structural changes in the $\mathrm{TH}$ accompanying dopamine-related cell death are proposed as important prodromes of PD (McKeown et al. 2008). The pathological symptoms are related to $\alpha$-synuclein aggregates within the intralaminar nuclei of the $\mathrm{TH}$ subregions. These lead to different levels of neuronal loss, which can result in varying levels of motor symptoms in monkey PD models (Smith et al. 2014; Halliday 2009). Halliday has stated that the TH plays a more central role in the pathophysiology of $\mathrm{PD}$, rather than just relaying pathological signals from the dopamine-deprived striatum through to the motor cortices (Halliday 2009). As $\alpha$ synuclein-a presynaptic neuronal protein-binds to iron, iron accumulation contributes to $\alpha$-synuclein aggregation and this is a major driver of PD pathogenesis (Ayton and Lei 2014; Li et al. 2018). The excessive iron depositions that generate oxidative stress are involved in mitochondrial damage, DNA damage, and lipid peroxidation. This ultimately leads to apoptosis (Jellinger 1999; Jiang et al. 2017; Li and Reichmann 2016) and contributes to the degeneration of dopaminergic neurons in PD (Xu et al. 2017). Therefore, investigations on iron accumulation within the $\mathrm{TH}$, especially within the anatomical subregions, may provide meaningful insights into PD pathogenesis.

Quantitative susceptibility mapping (QSM) to measure iron deposition has been demonstrated as a prominent method to evaluate cerebral abnormalities with higher sensitivity and specificity than conventional neuroimaging techniques such as T1, T2*-weighted contrast, and R2* relaxation rate mapping (Langkammer et al. 2016; Zhao et al. 2017; Murakami et al. 2015; Alkemade et al. 2017; Guan et al. 2017; Pietracupa et al. 2017). In PD brains, the magnetic susceptibility using QSM has been significantly increased in the SN and TH (Langkammer et al. 2016; Zhao et al. 2017; Murakami et al. 2015; Guan et al. 2017; Li et al. 2018; Pietracupa et al. 2017; Shahmaei et al. 2019). Most studies on PD are focused on impairment of the SN by iron accumulation, whereas the magnetic susceptibility of the TH using the QSM remains to be resolved using 3 $\mathrm{T}$ magnetic resonance imaging (MRI). This may be explained by the low susceptibility changes of approximately 5-11 parts per billion (ppb) within the TH in PD (Langkammer et al. 2016; Shahmaei et al. 2019), compared with susceptibility increases of 40-93 ppb in the SN. Moreover, variation in magnetic susceptibility has not yet been investigated within the intralaminar nuclei, although prior studies have found neuronal degeneration within the thalamic subregion (Smith et al. 2014; Halliday 2009). Subtle magnetic susceptibility changes in ultra-high-field MRI can be measured since the sensitivity of the magnetic susceptibility detection is directly proportional to the magnetic field strength of the MRI system (McKiernan and O'Brien 2017; van der Zwaag et al. 2016). Quantitative susceptibility mapping with high resolution using 7 T MRI also provides better visualization, by distinguishing substructures of the basal ganglia that were previously challenging to identify using low-field MRI (Ladd et al. 2018). Given these considerations, we speculate that evaluation of the iron deposition in the TH using $7 \mathrm{~T}$ MRI could be an informative biomarker for PD-related motor symptoms, which can be obtained using a non-invasive technique.

There are representative animal models to monitor the degeneration of dopaminergic neurons in the NSP based on neurotoxins, i.e. 6-hydroxydopamine (6OHDA) and 1-methyl-4-phenyl-1,2,3,6-tetrahydropyridine (MPTP) (Jagmag et al. 2015; Schober 2004). MPTP has enormous advantages as it can cross the blood-brain barrier and generate better results of representative PD symptoms such as $\alpha$-synuclein in the Lewy body and dopamine depletions (Jagmag et al. 2015). After MPTP administration to subjects, total iron accumulation in the cerebrum-an early PD feature-increases within 1 2 weeks, subsequently leading to $\alpha$-synuclein aggregates (Ayton and Lei 2014). The selective degeneration in the $\mathrm{TH}$, in 
addition to neuronal loss of the $\mathrm{SN}$, has been shown in MPTP-treated rats (Aymerich et al. 2006). In addition, monkey models have shown the loss of dopaminergic neurons through immunohistochemistry analysis, which also resulted in reduced dopamine activity as assessed by positron emission tomography (PET) with ${ }^{18} \mathrm{~F}-\mathrm{N}-(3-$ fluoro propyl)-2ß-carboxymethoxy-3ß-(4-iodophenyl) nortropane (18F-FP-CIT) (Seo et al. 2019; Blesa et al. 2012). Hence, this study was designed with cynomolgus monkeys to explore whether the assessment of susceptibility changes in the TH using QSM with a 7 T MRI system can be utilized as an early biomarker for PD diagnosis. In addition, PET with 18F-FP-CIT was employed to identify the relationship between the susceptibility changes and dopamine activity. Our findings exhibited thalamic changes in aberrant susceptibilities and dopaminergic activity, which distinguished an MPTP-treated monkey from a healthy control.

\section{Methods}

\section{Preparation of PD monkey by MPTP injection}

We used two age-matched adult, female cynomolgus monkeys (Macaca fascicularis) which were sourced from Zhaoqing Laboratory Animal Research Centre (Guangdong Province, China). They were maintained in individual indoor cages at the National Primate Research Center in the Korea Research Institute of Bioscience and Biotechnology (KRIBB). For a PD model, the MPTP was dissolved in saline $(0.45 \mathrm{mg} / \mathrm{kg}$ of body weight; SigmaAldrich, St. Louis, MO, USA), and injected intramuscularly into the left femoral region for 5 days. The total cumulative dose was $2.25 \mathrm{mg} / \mathrm{kg}$. The monkeys were monitored daily by a veterinarian (Y.B.J) and a caretaker (W.S.C). All procedures were approved by Korea Research Institute of Bioscience and Biotechnology Institutional Animal Care and Use Committee (Approval No. KRIBB-AEC-14018).

\section{MRI and PET data acquisitions}

A healthy monkey and an age-matched PD monkey were examined using $7 \mathrm{~T}$ MRI (Philips, Achieva, Best, Netherlands) with a 32-channel phased-array head coil (Nova Medical, MA, USA). The monkeys were first anesthetized with ketamine $(0.5 \mathrm{mg} / \mathrm{kg}$, intramuscular injection) and anesthesia was sustained with $2 \%$ isoflurane and $99.9 \%$ oxygen $(2 \mathrm{~L} / \mathrm{min})$. A blanket was used to maintain the body temperature. In addition, the pulse was monitored by peripheral gating instruments during the MRI scans. For the QSM, three-dimensional (3D) multi-gradient echo (mGRE) with flow compensation was acquired with second-order $B_{0}$ shimming. The angle between the monkey brain and the direction of $B_{0}$ was carefully adjusted for a reliable QSM reconstruction, since this angle tends to cause reconstruction errors in
(Haacke et al. 2015). The scan parameters of mGRE were as follows; $\mathrm{TR}=90 \mathrm{~ms}, \mathrm{TE}=4.6$ and $4.4 \mathrm{~ms}$ echo spacing, number of echoes $=4, F O V=100 \times 100 \mathrm{~mm}^{2}$, voxel size $=0.297 \times 0.297 \times 1 \mathrm{~mm}^{3}$, slab thickness $=52$ $\mathrm{mm}$ (whole brain coverage with the axial planes), flip angle $=22$ degrees, water-fat shift $=1.78 \mathrm{~Hz} /$ pixel (the lowest setting), number of average $=2$, total acquisition time $=49 \mathrm{~min} 33 \mathrm{~s}$. Subsequently, 3D T1-weighted images (T1w) were acquired to generate mask images for QSM with the following conditions: $\mathrm{TR}=9 \mathrm{~ms}, \mathrm{TE}=4.5$ $\mathrm{ms}$, inversion time $=1200 \mathrm{~ms}, \quad F O V=100 \times 100 \mathrm{~mm}^{2}$, voxel size $=0.297 \times 0.297 \times 1 \mathrm{~mm}^{3}$, slab thickness $=52$ $\mathrm{mm}$, flip angle $=6$ degrees, water-fat shift $=4.68 \mathrm{~Hz} /$ pixel, number of average $=10$, total acquisition time $=$ $26 \mathrm{~min} 58 \mathrm{~s}$.

All PET images were acquired using a PET/computed tomography (CT) scanner (nanoScan PET-CT, Mediso Ltd., Budapest, Hungary) to evaluate dopamine transporter activities with 18F-FP-CIT (Kazumata et al. 1998). The drug was obtained from a commercial company (DuChemBio Co., Ltd., Daejeon, Korea). Anesthesia was induced in the same way as was induced during MRI. A CT scan was implemented for attenuation correction, and $185 \mathrm{MBq}$ of $18 \mathrm{~F}-\mathrm{FP}-\mathrm{CIT}$ mixed in $1.5 \mathrm{~mL}$ saline was intravenously injected into the saphenous vein. PET acquisition with $400 \times 400$ matrix size in the list mode was concomitantly started with the injection for $120 \mathrm{~min}$. The dynamic sequences were composed of a total 39-frame sets: $15 \mathrm{~s} \times 8,30 \mathrm{~s} \times 6,60 \mathrm{~s} \times 5,120 \mathrm{~s} \times 5$, $300 \mathrm{~s} \times 10$, and $600 \mathrm{~s} \times 5$ frames.

\section{Reconstruction and registration of susceptibility and dopamine activity}

The 3D-mGRE images were used to reconstruct QSM images using STI Suite (Guan et al. 2017). The reconstruction steps consisted of 1) Laplacian phase unwrapping, 2) iterative harmonic phase removal using the Laplacian operator (iHARPERELLA) for background phase removal, and 3) least-square orthogonal and righttriangular decomposition (LSQR) for field-tosusceptibility inversion. For background removal, mask images of cerebral parenchyma areas only were generated using the automated brain extraction tool (BET, http://www.fmrib.ox.ac.uk/fsl) (Jenkinson et al. 2012). All reconstructed QSM images were registered to the templated-primate brain images, named INIA19 (Rohlfing et al. 2012), using the 3D Slicer (https://www.slicer.org/) (Fedorov et al. 2012) to identically observe the magnetic susceptibility changes. Using NeuroMap classified as subanatomical regions (Rohlfing et al. 2012), each anatomical region of the NSPs (the $\mathrm{SN}, \mathrm{GP}, \mathrm{PT}, \mathrm{CN}$, and $\mathrm{TH}$ ) was semi-automatically segmented for mask images (Fig. 1a), which were generated by MATLAB (Mathworks, MA, USA). Subsequently, the cerebrospinal fluid (CSF) in the 


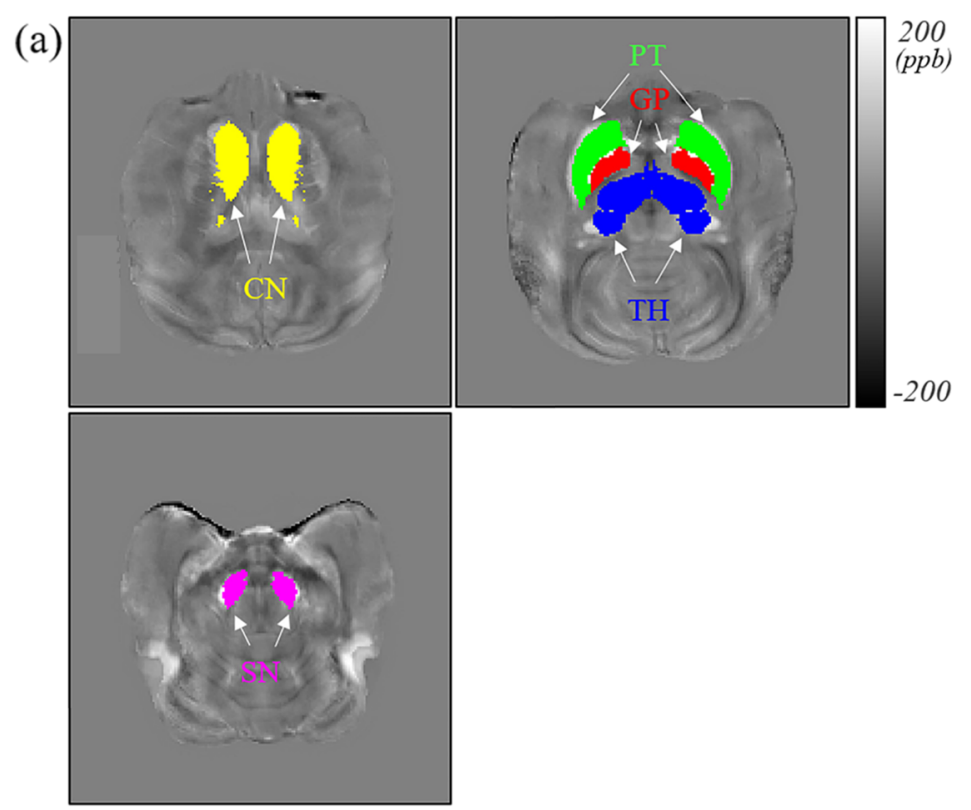

(b)
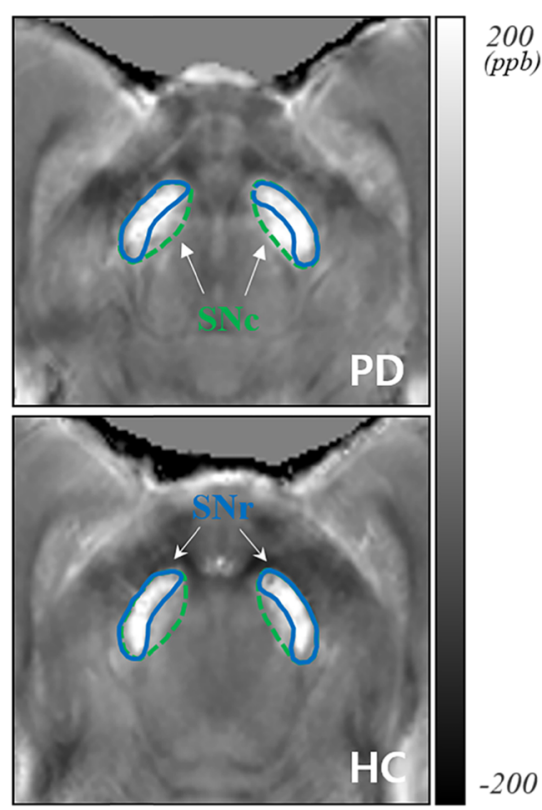

Fig. 1 Cerebral-masked regions of the NSP and the SN. a Each cerebral region of the NSP overlaid on reconstructed QSM images. b The SN divided into two regions, the SNc and SNr. NSP = nigrostriatal pathway; $C N=$ caudate nucleus; $\mathrm{PT}=$ putamen; $\mathrm{GP}=$ globus pallidus; $\mathrm{TH}=$ thalamus; SN = substantia nigra; HC = healthy control; PD = Parkinson's disease; SNc = SN pars compacta; SNr = SN pars reticulate

QSMs was subtracted by the masked CSF regions that were extracted from 3D T1w using the BET. This is due to the process generating non-uniform signals of cerebrospinal fluid flow and white matter anisotropy in regions adjacent to the ventricles (Liu et al. 2018). Lastly, the TH was divided into three subregions using the NeuroMapthe ventral, intralaminar, and medial (Halliday 2009). The magnetic susceptibility in all the masked regions of the PD model, the NSP and the thalamic subregions, were quantified as mean values and compared with those of a healthy control.

The acquired PET images were reconstructed using a three-dimensional ordered subsets expectation maximization algorithm (Tera-Tomo, Mediso Ltd., Hungary), and postprocessed using syngo VG51C software (Siemens Medical Solutions, Erlangen, Germany) to visualize multiplanar fusion images. Reconstructed voxel values in each frame were reported in standard uptake value from body weight and corrected for radioactive decay from the time of injection. The voxel dimensions were $0.4 \times 0.4 \times 0.4 \mathrm{~mm}^{3}$. In total, 39 frames sets obtained after 18F-FP-CIT injection, the final three frames relevant to the $90-120 \mathrm{~min}$ time point were used for dynamic analysis, as the specific striatal uptake had reached to a maximal value in the baseline. The monkeytemplated dataset and the volumetric mask of the NSPsthe INIA19-T1 images and the identical mask images used in the QSM, respectively-were manually fed into the IRW (Inveon Research Workplace, Siemens Medical Solutions, USA) software. The T1-templated images were utilized to perform the manual and rigid registration of the PET images.
The binding potential (BP) relative to the concentration of non-displaceable radioligand in each region of the volumetric NSP masks was normalized by using the Reference Logan Plot, where the reference region is the cerebellum (Logan et al. 1996). Subsequently, the BP values of the 3D NSP regions in the healthy control and PD models were averaged and compared between the objects.

\section{Results}

The averaged QSM values were measured to compare iron deposition at each anatomical region in the NSPs. Compared with control, magnetic susceptibility values in the PD model tended to increase (Fig. 2) except in the globus pallidus (decreased 18.89\%, $\mathrm{PD}=139.09 \mathrm{ppb}$ versus $\mathrm{HC}=170.02 \mathrm{ppb}$ ). The averaged susceptibility changes in $\mathrm{CN}, \mathrm{PT}, \mathrm{TH}$, and $\mathrm{SN}$ were increased from $38 \%$ to $54 \%$ in the PD model $(\mathrm{CN}=38.76 \%, \mathrm{PT}=24.29 \%, \mathrm{TH}=$ $53.81 \%$, and $\mathrm{SN}=54.81 \%$ ) when they were compared with the $\mathrm{HC}$. The remarkable alteration of the magnetic susceptibilities in the PD was shown in the TH at 53.81\% (39.40 ppb in PD model and $18.20 \mathrm{ppb}$ in control) and in the $\mathrm{SN}$ at $54.81 \%$ (83.19 ppb in PD model and $37.60 \mathrm{ppb}$ in control).

The TH's subregions were scrutinized to identify susceptibility changes of the PD model. The magnetic susceptibilities were substantially increased from $20 \%$ to $70 \%$ (ventral $=57.97 \%$, intralaminar $=70.78 \%$, and medial $=20.83 \%$ ). The intralaminar part of the PD model showed 4.42 times altered susceptibility when compared with $\mathrm{HC}(\mathrm{PD}=15.63 \mathrm{ppb}, \mathrm{HC}=-4.57 \mathrm{ppb}$, Fig. 3). The 


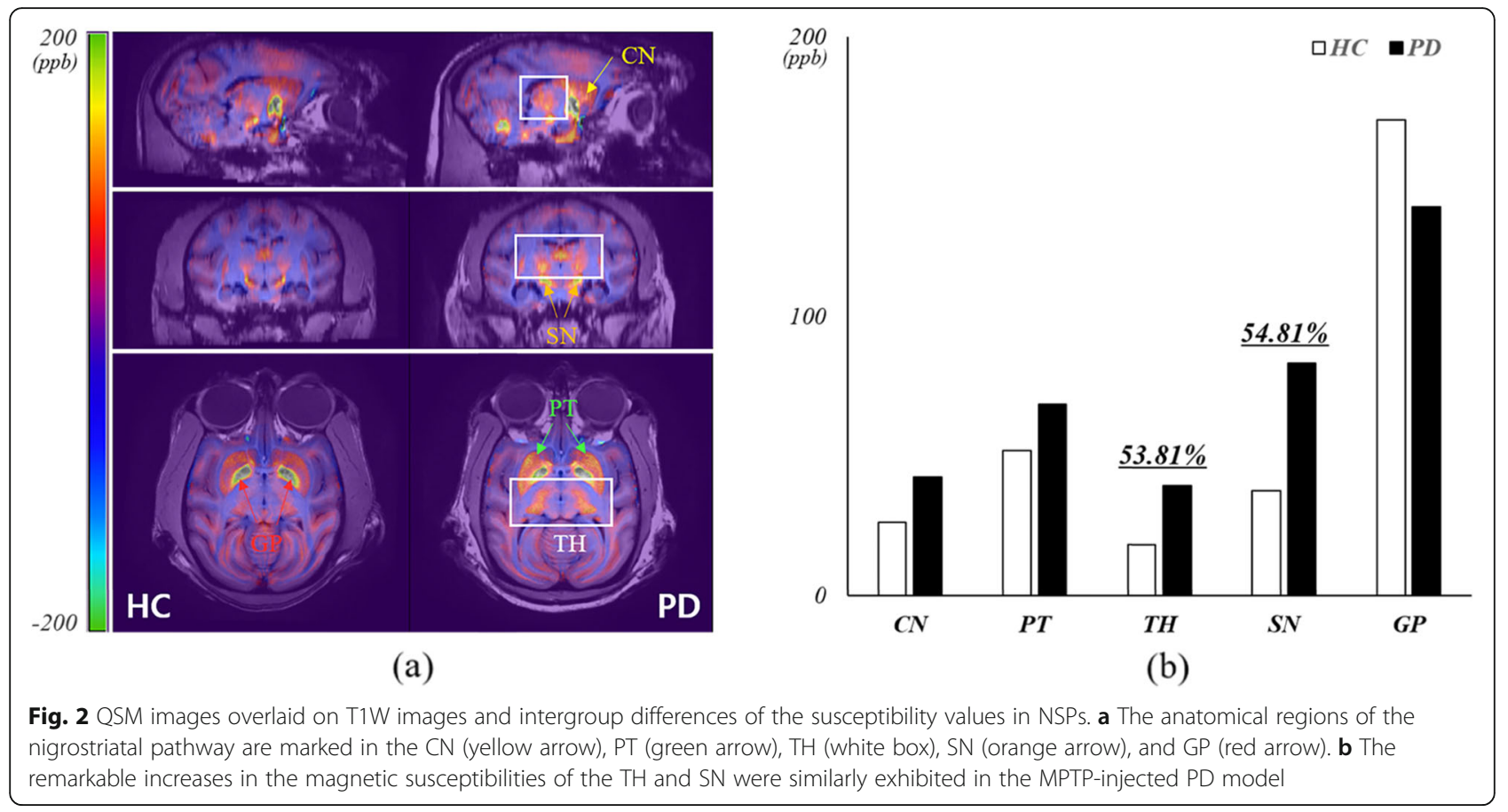

(a)

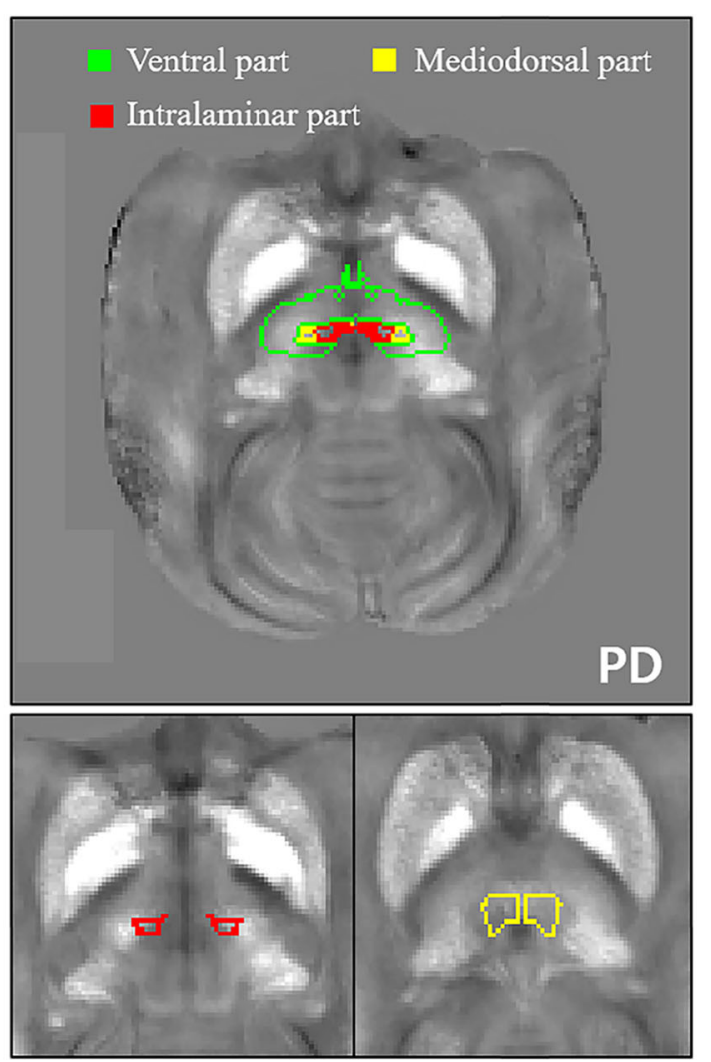

(b) 50

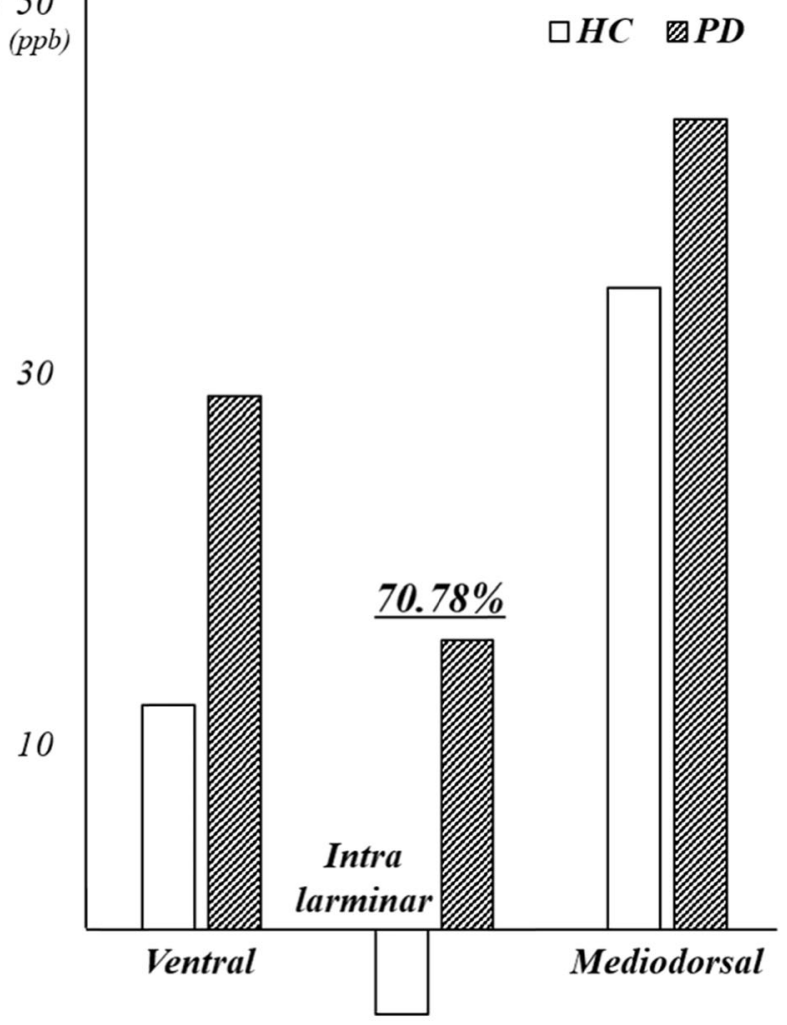

Fig. 3 Quantitative susceptibility values of thalamic subregions, and their differences in PD versus control monkey. a The region of interest of the ventral, intralaminar, and mediodrosal part is exhibited. $\mathbf{b}$ Compared with the healthy control, the relative proportion of the iron depositions was increased in the subthalamic regions of the PD animal, especially a considerable elevation in the intralaminar region than the other nuclei 
susceptibility results of all thalamic regions were summarized in Table 1. In addition, the SN was divided into two regions (Fig. 1b), the $\mathrm{SN}$ pars compacta $(\mathrm{SNc})$ and $\mathrm{SN}$ pars reticulata $(\mathrm{SNr})$. The $\mathrm{SNc}$ is a region wellknown to show early abnormalities PD (Ayton and Lei 2014; Guan et al. 2017; Lehéricy et al. 2014). The iron deposition ratio in the PD model was observed to be higher in the SNc (about $40.50 \%$ increase, $114.00 \mathrm{ppb}$ in $\mathrm{PD}$ versus $67.83 \mathrm{ppb}$ in $\mathrm{HC}$ ) than in the $\mathrm{SNr}(149.11 \mathrm{ppb}$ in PD versus $150.46 \mathrm{ppb}$ in $\mathrm{HC}$ ). This result within the $\mathrm{SN}$ supports the findings from the previous PD studies (Guan et al. 2017; Ayton and Lei 2014; Lehéricy et al. 2014).

The BP values using PET were evaluated to ascertain the relationship between susceptibility changes and dopaminergic activity on in NSP regions. In the PD model, the BP of all NSPs was considerably reduced from 77 to $97 \%$, when compared with the $\mathrm{HC}$ (Fig. 4). A striking decrease was exhibited in the $\mathrm{TH}(-97.51 \%, \mathrm{PD}=-0.00838 \mathrm{BP}$ versus $\mathrm{HC}=0.3369 \mathrm{BP})$ and also in the $\mathrm{SN}(-92.48 \%$, $\mathrm{PD}=0.0359 \mathrm{BP}$ versus $\mathrm{HC}=0.4776 \mathrm{BP}$ ).

\section{Discussion}

We employed the QSM technique to quantify magnetic susceptibility differences in subcortical regions of the nigrostriatal pathway. We demonstrated an overall elevation in iron depositions in the cynomolgus monkey with PD (Fig. 2), with iron depositions increased about 2.2 times in the thalamus, which was a similar level to the substantia nigra. In addition, a substantial susceptibility change was exhibited in the intralaminar part of the $\mathrm{TH}$ (about $70.78 \%$ increase, Fig. 3). Moreover, we found that dopamine neuronal activity, measured using PET, was considerably decreased in the abnormal regions of susceptibility in the PD animal. These results support the hypothesis that iron depositions are negatively correlated to dopamine activity in PD. A proposed mechanism for this effect would be that increased iron depositions in the thalamus cause damage to glutaminergic inputs and dopaminergic neurons, leading to motor malfunction due to synaptic dysfunction.

Noteworthy findings include, firstly, that the remarkable susceptibility change in the thalamus $(53.81 \%)$ is

Table 1 Averaged QSM values taken for each anatomical region of the thalamus. The intralaminar part of the PD animal showed a considerable increase more than the other part of the thalamic subregions in comparison with that of the healthy control

\begin{tabular}{lll}
\hline & $\mathrm{HC}[\mathrm{ppb}]$ & $\mathrm{PD}[\mathrm{ppb}]$ \\
\hline Ventral part & 12.09 & 28.77 \\
Intralaminar part & -4.57 & 15.63 \\
Mediodorsal part & 34.61 & 43.71 \\
\hline
\end{tabular}

Magnetic susceptibility values are given in part per billion (ppb) similar to that of the SN (54.81\%), secondly, that the magnetic susceptibility in the $\mathrm{SNc}$ is increased in PD model relative to the $\mathrm{SNr}$. The $\mathrm{SNc}$ has been reported as a region sensitive to iron depositions in the early stage of PD (Langkammer et al. 2016; Zhao et al. 2017; Murakami et al. 2015; Hodaie et al. 2007; Guan et al. 2017; Ayton and Lei 2014; Lehéricy et al. 2014). The iron overload overwhelms the antioxidant capacity of the tissue, resulting in oxidative stress (Jellinger 1999; Jiang et al. 2017; Li and Reichmann 2016). Dopaminergic neurons in the SNc are sensitive to these effects, subsequently leading to cell dysfunction and ultimately cell loss (Jiang et al. 2017). Through immunofluorescence, the relationship between iron accumulation and the loss of dopaminergic neurons has been confirmed in the SNc of MPTP-injected models (Kowall et al. 2000; You et al. 2015), which is consistent with our observations. We reinforce that the $\mathrm{SN}$ is one of the primary anatomical structures that is used for PD diagnosis. Interestingly, Halliday had stated that early features of PD pathology are closely associated with thalamic alterations in concert with dopamine depletion, which reliably support our results as an initial sign of PD (Halliday 2009). Several studies have shown that selective structural changes in the thalamus of PD are significantly different from that of healthy controls (Lee et al. 2011; Garg et al. 2015; McKeown et al. 2008). However, assessment of the susceptibility changes using QSM has been proven to be more sensitive than morphological changes at identifying cerebral abnormalities in diseases such as Parkinson's and Alzheimer's diseases (Murakami et al. 2015; Kim et al. 2017; Liu et al. 2015; Wang and Liu 2015). The increased magnetic susceptibility using QSM has been exhibited in the thalamus of PD patients (Langkammer et al. 2016; Li et al. 2018; Shahmaei et al. 2019) and this is in line with the observations in this study. Interestingly, decreased values have been shown in the elderly using QSM and the field-dependent relaxation rate increase method (Pfefferbaum et al. 2009; Bilgic et al. 2012). These results mean that the increased susceptibility in the thalamus could be a major diagnostic biomarker for early PD pathology. However, additional studies are needed to reveal the relationship between increased susceptibilities and structural changes in the thalamus.

Our results also demonstrate the substantial alteration in susceptibility within the intralaminar region of the thalamus (about $70.78 \%$ increased), which is consistent with a former study that detected excessive iron deposits within Lewy neurites in the thalamic subregion of human PD (Brooks and Halliday 2009). The thalamus modulates direct and indirect movement effects by synapsing on the glutamatergic and GABAergic neurons (Hodaie et al. 2007; Guan et al. 2017). In particular, the 

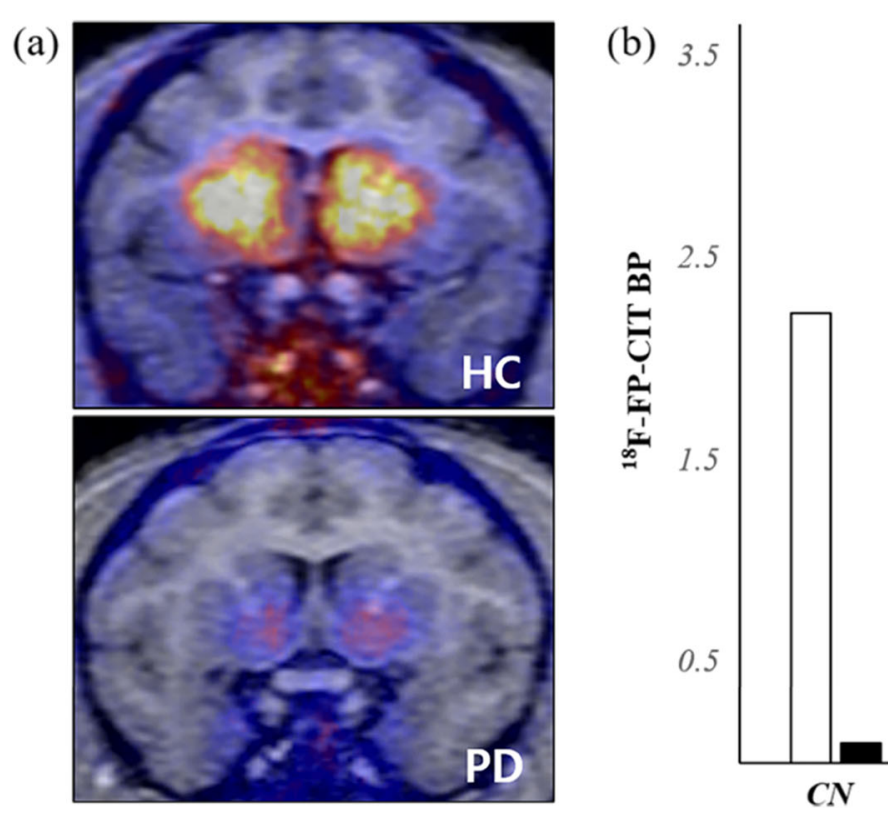

Fig. 4 Dopamine activity of a healthy control and PD model using PET. a PET images overlaid with the T1-weighted image. b In the PD animal, the substantial decreases in dopamine activities are exhibited in the nigrostriatal pathway compared with the control. BP = binding potential

intralaminar nuclei provide major glutamatergic inputs to the whole striatum without dopamine regulation (Halliday 2009). Of the representative PD symptoms, the tremor in resting-state is especially related to subtle thalamic abnormalities (Halliday 2009). It is mainly caused by the loss of dopaminergic neurons (Magrinelli et al. 2016) and concentrated on the intralaminar part of the thalamus in the diseased individuals (McKeown et al. 2008; Brooks and Halliday 2009; Villalba et al. 2014). Parker et al. have discovered that the excitatory postsynaptic strengths of the thalamostriatal direct pathway, which accompany the higher activation of corticostriatal indirect tracts, are decreased in 6OHDA-injected PD models, while healthy rodents show the reversed mechanism (Parker et al. 2016). Although there are differences in the injected drug and animal type compared with our study, nerve terminals to the striatum have been largely shown to degenerate in the MPTP-injected monkey (Blesa et al. 2010). These indicate that iron accumulation in the thalamus of PD may be associated with the manipulation of thalamic input excitability, leading to the progressive and severe motor symptoms. Consequently, the increased susceptibility values in the thalamus could be used in a non-invasive manner to diagnose PD with tremor symptoms. However, extensive research is required to validate the correlation between the behavioral failure in PD and the iron depositions.

In the present study, about two-folded magnetic susceptibility increases were shown in the ventral nuclei of the thalamic subregion. As the ventral regions control motor cortices by processing signals from the basal ganglia and cerebellum (George and Das 2019), the abnormality in magnetic susceptibility may contribute to PD motor symptoms such as bradykinesia and rigidity. The influence on the ventral region should be distinguished from PD disorders induced by abnormalities in the intralaminar nuclei. In the substantia nigra, the susceptibility changes are consistent with previous studies in PD (Langkammer et al. 2016; Zhao et al. 2017; Murakami et al. 2015; Guan et al. 2017; Pietracupa et al. 2017). However, in these studies, the results within the $\mathrm{TH}$ were cautiously interpreted, since the number of PD prototypes is insufficient to generalize cerebral abnormalities related to PD symptoms. Further studies are needed with larger non-human primate models to verify our findings. Subsequently, immunohistochemical methods are necessary to identify the iron depositions within the thalamus of the MPTP-injected PD model. It is possible that the drug used to induce PD, MPTP and 6OHDA, may result in differences between the models. The motor-activated pathways changed in the 6OHDAinjection models (Parker et al. 2016) may differ to the excitatory synaptic responses induced by MPTP, so additional studies are needed. Lastly, our results also show susceptibility alterations in the caudate nucleus, globus pallidus, and the putamen. The interpretation of the results remains controversial, since iron concentrations in the striatum are significantly increased with age in humans and monkeys (Bilgic et al. 2012; Cass et al. 2007). Additional studies are needed to distinguish whether the susceptibility distribution in the corresponding regions is a result of PD or aging. 


\section{Conclusion}

In this study, we demonstrated increased magnetic susceptibilities of the thalamus in the monkey MPTP injection model of PD, using QSM with a $7 \mathrm{~T}$ MRI system. Iron deposition was negatively correlated to dopamine activity in the PD animal. The remarkable susceptibility changes in the thalamus may manifest damage to dopaminergic neurons, thereby leading to motor function failure as a result of synaptic dysfunction. Therefore, QSM with 7 T MRI could be utilized as an initial major diagnostic biomarker for PD-related motor symptoms.

\begin{abstract}
Abbreviations
18F-FP-CIT: ${ }^{18} \mathrm{~F}-\mathrm{N}$-(3-fluoro propyl)-2ß-carboxymethoxy-3ß-(4-iodophenyl) nortropane; 6OHDA: 6-Hydroxydopamine; BP: Binding potential; CN: Caudate nucleus; CSF: Cerebrospinal fluid; GP: Globus pallidus; mGRE: Multi-gradient echo; MPTP: 1-Methyl-4-phenyl-1,2,3,6-tetrahydropyridine; MRI: Magnetic resonance imaging; NSP: Nigrostriatal pathway; PD: Parkinson's disease; PET: Positron emission tomography; ppb: Parts per billion; PT: Putamen; QSM: Quantitative susceptibility mapping; SN: Substantia nigra; T1 w: T1weighted images; TH: Thalamus
\end{abstract}

\section{Acknowledgements}

Not applicable.

\section{Funding}

This study was supported by the National Research Council of Science \& Technology (NST) grant from the government of Korea (MSIP) (No. CPS-1802-KBSI) and by the Korea Basic Science Institute grant (C39134).

\section{Availability of data and materials}

The datasets used in this study are available on a reasonable request from the corresponding author.

\section{Authors' contributions}

All authors read and approved the final manuscript.

\section{Competing interests}

The authors declare that they have no competing interests.

\section{Author details}

${ }^{1}$ Department of Bio-Analytical Science, University of Science and Technology, 217, Gajeong-ro, Yuseong-gu, Daejeon, Republic of Korea. ${ }^{2}$ Bioimaging Research Team, Korea Basic Science Institute, 162, Yeongudanji-ro, Ochang-eup, Cheongwon-gu, Cheongju-si, Chungcheongbuk-do, Republic of Korea. ${ }^{3}$ National Primate Research Center, Korea Research Institute of Bioscience and Biotechnology, 30, Yeongudanji-ro, Ochang-eup, Cheongwon-gu, Cheongju-si, Chungcheongbuk-do, Republic of Korea.

Received: 5 November 2019 Accepted: 18 December 2019 Published online: 29 December 2019

\section{References}

Albin $\mathrm{RL}$, Young $\mathrm{AB}$, Penney JB. The functional anatomy of basal ganglia disorders. Trends Neurosci. 1989;12:366-75.

Alkemade A, de Hollander G, Keuken MC, Schäfer A, Ott DV, Schwarz J, Weise D, Kotz SA, Forstmann BU. Comparison of T2*-weighted and QSM contrasts in Parkinson's disease to visualize the STN with MRI. PLoS One. 2017;12: e0176130.

Aymerich MS, Barroso-Chinea P, Perez-Manso M, Munoz-Patino AM, Moreno-Igoa M, Gonzalez-Hernandez T, Lanciego JL. Consequences of unilateral nigrostriatal denervation on the thalamostriatal pathway in rats. Eur J Neurosci. 2006;23:2099-108.

Ayton S, Lei P. Nigral iron elevation is an invariable feature of Parkinson's disease and is a sufficient cause of neurodegeneration. BioMed Res Int. 2014;2014: 581256. https://doi.org/10.1155/2014/581256.
Bilgic B, Pfefferbaum A, Rohlfing T, Sullivan EV, Adalsteinsson E. MRI estimates of brain iron concentration in normal aging using quantitative susceptibility mapping. Neuroimage. 2012;59:2625-35.

Blesa J, Juri C, Collantes M, Peñuelas I, Prieto E, Iglesias E, Martí-Climent J, Arbizu J, Zubieta JL, Rodríguez-Oroz MC. Progression of dopaminergic depletion in a model of MPTP-induced parkinsonism in non-human primates. An 18FDOPA and 11C-DTBZ PET study. Neurobiol Dis. 2010;38:456-63.

Blesa J, Pifl C, Sanchez-Gonzalez MA, Juri C, Garcia-Cabezas MA, Adanez R, Iglesias E, Collantes M, Penuelas I, Sanchez-Hernandez JJ, Rodriguez-Oroz MC, Avendano C, Hornykiewicz O, Cavada C, Obeso JA. The nigrostriatal system in the presymptomatic and symptomatic stages in the MPTP monkey model: a PET, histological and biochemical study. Neurobiol Dis. 2012;48:79-91.

Brooks D, Halliday GM. Intralaminar nuclei of the thalamus in Lewy body diseases. Brain Res Bull. 2009;78:97-104.

Cass WA, Grondin R, Andersen AH, Zhang Z, Hardy PA, Hussey-Andersen LK, Rayens WS, Gerhardt GA, Gash DM. Iron accumulation in the striatum predicts aging-related decline in motor function in rhesus monkeys. Neurobiol Aging. 2007;28:258-71.

DeLong MR. Primate models of movement disorders of basal ganglia origin. Trends Neurosci. 1990;13:281-5.

Fedorov A, Beichel R, Kalpathy-Cramer J, Finet J, Fillion-Robin J-C, Pujol S, Bauer C, Jennings D, Fennessy F, Sonka M. 3D slicer as an image computing platform for the quantitative imaging network. Magn Reson Imag. 2012;30:1323-41.

Garg A, Appel-Cresswell S, Popuri K, McKeown MJ, Beg MF. Morphological alterations in the caudate, putamen, pallidum, and thalamus in Parkinson's disease. Front Neurosci. 2015;9:101.

George, K, Das, J. M (2019) Neuroanatomy, Thalamocortical Radiations. StatPearls Publishing. https:/muw.ncbi.n/m.nih.gov/books/NBK546699/. Accessed 05 Dec 2019.

Guan X, Xuan M, Gu Q, Huang P, Liu C, Wang N, Xu X, Luo W, Zhang M. Regionally progressive accumulation of iron in Parkinson's disease as measured by quantitative susceptibility mapping. NMR Biomed. 2017;30(4): e3489.

Haacke EM, Liu S, Buch S, Zheng W, Wu D, Ye Y. Quantitative susceptibility mapping: current status and future directions. Magn Reson Imaging. 2015:33:1-25.

Halliday GM. Thalamic changes in Parkinson's disease. Parkinsonism Relat D. 2009; 15:S152-5.

Hodaie M, Neimat JS, Lozano AM. The dopaminergic nigrostriatal system and Parkinson's disease: molecular events in development, disease, and cell death, and new therapeutic strategies. Neurosurg. 2007;60:17-28.

Jagmag SA, Tripathi N, Shukla SD, Maiti S, Khurana S. Evaluation of models of Parkinson's disease. Front Neurosci. 2015;9:503.

Jellinger KA. The role of iron in neurodegeneration: prospects for pharmacotherapy of Parkinson's disease. Drugs Aging. 1999;14:115-40.

Jenkinson M, Beckmann CF, Behrens TE, Woolrich MW, Smith SM. FSL. Neuroimage. 2012;62:782-90.

Jiang H, Wang J, Rogers J, Xie J. Brain Iron metabolism dysfunction in Parkinson's disease. Mol Neurobiol. 2017;54:3078-101.

Kazumata K, Dhawan V, Chaly T, Antonini A, Margouleff C, Belakhlef A, Neumeyer J, Eidelberg D. Dopamine transporter imaging with fluorine-18-FPCIT and PET. J Nucl Med. 1998;39:1521-30.

Kim H-G, Park S, Rhee HY, Lee KM, Ryu C-W, Rhee SJ, Lee SY, Wang Y, Jahng G-H. Quantitative susceptibility mapping to evaluate the early stage of Alzheimer's disease. Neurolmage Clin. 2017;16:429-38.

Kowall NW, Hantraye P, Brouillet E, Beal MF, McKee AC, Ferrante RJ. MPTP induces alpha-synuclein aggregation in the substantia nigra of baboons. Neuroreport. 2000;11:211-3.

Ladd ME, Bachert P, Meyerspeer M, Moser E, Nagel AM, Norris DG, Schmitter S, Speck O, Straub S, Zaiss M. Pros and cons of ultra-high-field MRI/MRS for human application. Prog Nucl Magn Reson Spectrosc. 2018;109:1-50.

Langkammer C, Pirpamer L, Seiler S, Deistung A, Schweser F, Franthal S, Homayoon N, Katschnig-Winter P, Koegl-Wallner M, Pendl T. Quantitative susceptibility mapping in Parkinson's disease. PLoS One. 2016;11:e0162460.

Lee SH, Kim SS, Tae WS, Lee SY, Choi JW, Koh SB, Kwon DY. Regional volume analysis of the Parkinson disease brain in early disease stage: gray matter, white matter, striatum, and thalamus. AJNR Am J Neuroradiol. 2011;32:682-7.

Lehéricy S, Bardinet E, Poupon C, Vidailhet M, François C. 7 tesla magnetic resonance imaging: a closer look at substantia nigra anatomy in Parkinson's disease. Move Disord. 2014;29:1574-81.

Li DT, Hui ES, Chan Q, Yao N, Chua S, McAlonan GM, Pang SY, Ho S, Mak HK. Quantitative susceptibility mapping as an indicator of subcortical and limbic 
iron abnormality in Parkinson's disease with dementia. Neurolmage Clin. 2018:20:365-73.

Li K, Reichmann H. Role of iron in neurodegenerative diseases. J Neural Transm (Vienna). 2016;123:389-99.

Liu C, Wei H, Gong NJ, Cronin M, Dibb R, Decker K. Quantitative susceptibility mapping: contrast mechanisms and clinical applications. Tomography. 2015; $1: 3-17$.

Liu Z, Spincemaille P, Yao Y, Zhang Y, Wang Y. MEDI+0: morphology enabled dipole inversion with automatic uniform cerebrospinal fluid zero reference for quantitative susceptibility mapping. Magn Reson Med. 2018;79:2795-803.

Logan J, Fowler JS, Volkow ND, Wang G-J, Ding Y-S, Alexoff DL. Distribution volume ratios without blood sampling from graphical analysis of PET data. J Cereb Blood Flow Metab. 1996;16:834-40.

Magrinelli F, Picelli A, Tocco P, Federico A, Roncari L, Smania N, Zanette G, Tamburin S. Pathophysiology of motor dysfunction in Parkinson's disease as the rationale for drug treatment and rehabilitation. Parkinsons Dis. 2016;2016: 9832839. https://doi.org/10.1155/2016/9832839.

McKeown MJ, Uthama A, Abugharbieh R, Palmer S, Lewis M, Huang X. Shape (but not volume) changes in the thalami in Parkinson disease. BMC Meurol. 2008;8:8.

McKiernan EF, O'Brien JT. 7T MRI for neurodegenerative dementias in vivo: a systematic review of the literature. J Neurol Neurosurg Psychiatry. 2017;88: 564-74.

Murakami Y, Kakeda S, Watanabe K, Ueda I, Ogasawara A, Moriya J, Ide S, Futatsuya K, Sato T, Okada K, Uozumi T, Tsuji S, Liu T, Wang Y, Korogi Y. Usefulness of quantitative susceptibility mapping for the diagnosis of Parkinson disease. AJNR Am J Neuroradiol. 2015;36:1102-8.

Parker PR, Lalive AL, Kreitzer AC. Pathway-specific remodeling of thalamostriatal synapses in Parkinsonian mice. Neuron. 2016;89:734-40.

Pfefferbaum A, Adalsteinsson E, Rohlfing T, Sullivan EV. MRI estimates of brain iron concentration in normal aging: comparison of field-dependent (FDRI) and phase (SWI) methods. Neuroimage. 2009:47:493-500.

Pietracupa S, Martin-Bastida A, Piccini P. Iron metabolism and its detection through MRI in parkinsonian disorders: a systematic review. Neurol Sci. 2017; 38:2095-101.

Rohlfing T, Kroenke CD, Sullivan EV, Dubach MF, Bowden DM, Grant K, Pfefferbaum A. The INIA19 template and NeuroMaps atlas for primate brain image parcellation and spatial normalization. Front Neuroinformat. 2012;6:27.

Schober A. Classic toxin-induced animal models of Parkinson's disease: 6-OHDA and MPTP. Cell Tissue Res. 2004;318:215-24.

Seo J, Lee Y, Kim BS, Park J, Yang S, Yoon H-J, Yoo J, Park HS, Hong J-J, Koo B-S. A non-human primate model for stable chronic Parkinson's disease induced by MPTP administration based on individual behavioral quantification. $J$ Neurosci Methods. 2019;311:277-87.

Shahmaei V, Faeghi F, Mohammdbeigi A, Hashemi H, Ashrafi F. Evaluation of iron deposition in brain basal ganglia of patients with Parkinson's disease using quantitative susceptibility mapping. Eur J Radiol Open. 2019;6:169-74.

Smith Y, Galvan A, Ellender TJ, Doig N, Villalba RM, Huerta-Ocampo I, Wichmann T, Bolam JP. The thalamostriatal system in normal and diseased states. Front Syst Neurosci. 2014;8:5.

van der Zwaag W, Schafer A, Marques JP, Turner R, Trampel R. Recent applications of UHF-MRI in the study of human brain function and structure: a review. NMR Biomed. 2016;29:1274-88.

Villalba RM, Wichmann T, Smith Y. Neuronal loss in the caudal intralaminar thalamic nuclei in a primate model of Parkinson's disease. Brain Struct Funct. 2014;219:381-94.

Wang Y, Liu T. Quantitative susceptibility mapping (QSM): decoding MRI data for a tissue magnetic biomarker. Magn Reson Med. 2015;73:82-101.

Xu H, Wang Y, Song N, Wang J, Jiang H, Xie J. New progress on the role of glia in iron metabolism and iron-induced degeneration of dopamine neurons in Parkinson's disease. Front Mol Neurosci. 2017;10:455.

You LH, Li F, Wang L, Zhao SE, Wang SM, Zhang LL, Zhang LH, Duan XL, Yu P, Chang YZ. Brain iron accumulation exacerbates the pathogenesis of MPTPinduced Parkinson's disease. Neuroscience. 2015;284:234-46.

Zhao X, An H, Liu T, Shen N, Bo B, Zhang Z, Weng P, Chen M, Pei M, Wang Y. Quantitative susceptibility mapping of the substantia nigra in Parkinson's disease. Appl Magn Reson. 2017;48:533-44.

\section{Publisher's Note}

Springer Nature remains neutral with regard to jurisdictional claims in published maps and institutional affiliations.

\section{Submit your manuscript to a SpringerOpen ${ }^{\circ}$ journal and benefit from:}

- Convenient online submission

- Rigorous peer review

- Open access: articles freely available online

- High visibility within the field

- Retaining the copyright to your article

Submit your next manuscript at $\boldsymbol{\nabla}$ springeropen.com 